\title{
Reconstructed from skin and bone
}

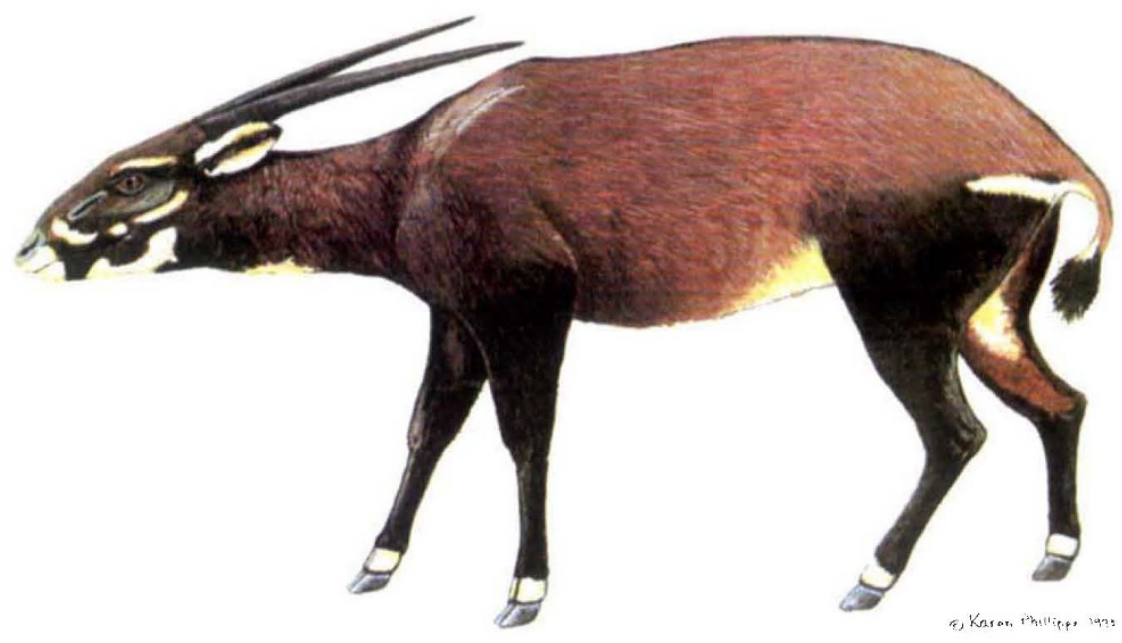

JUST when one might think that people have looked behind every tree on the planet for hitherto undescribed mammals, one emerges from the forests of Vietnam. In a classic piece of old-fashioned zoology, V. V. Dung et al. (page 443 of this issue) describe a new species of bovid solely on the basis of local hunting trophies skulls, teeth and skins. The picture above is an artist's reconstruction, and science has yet to scrutinize a living specimen of Pseudoryx nghetinhensis. A few hundred animals may exist in the montane forests of the Vietnamese-Laotian border, and its status must be considered as endangered.

The case of another rare even-toed ungulate, the Chacoan peccary (Catagonus wagneri), may be a guide to its fate. This pig-like animal had been known exclusively from fossils, until a living population was discovered in the Chaco region on the borders of Bolivia, Paraguay and Argentina in the 1970s. Until the arrival of Pseudoryx, the peccary was the latest and last large mammal to have been described - but hunting and habitat destruction could wipe it out before this century closes.

Others are even less lucky: the gazelle Gazella ruffina is known only from skins garnered from nineteenth-century Algerian souks. Undeterred by the subsequent non-appearance of $G$. ruffina, Dung and colleagues intend to hunt for living specimens of Pseudoryx. The animal gets its name from the oryx, which has similar long, graceful horns. But wild populations of the Arabian oryx, Oryx leucoryx, were wiped out in the 1970s, and the species was saved only by captive breeding.

The benign hunt for Pseudoryx should have a similar aim - to rescue the animal and transfer it to a safe haven. Indeed, the animal could have chosen better places to emerge from hiding than Indochina. This is the haunt of another shy bovid, the kouprey (Bos sauveli), described in 1937. It numbered about 1,000 animals in 1940, but was down to 100 in 1969. "Since its discovery," writes Ronald Nowak (in Walker's Mammals of the World, 5th edn, Johns Hopkins University Press, 1991), "its limited range has been the scene of almost constant human warfare." The kouprey was reported to be hanging on, albeit grimly, in 1986. Reports of its demise will be neither an exaggeration nor, sadly, a surprise.

H.G.

precisely general relativity, although it is closely related to both general relativity and the low-energy limit of superstring theories. This theory, unlike general relativity, has dynamics in two dimensions.

The second stratagem used by Callan et al. is to study extreme charged black holes, that is, black holes whose charge is equal in magnitude to their mass (in 'natural units' where Planck's constant, Newton's constant and the speed of light are all set equal to unity). Such black holes have zero temperature, and hence are stable against evaporative particle creation, ignoring charged particle creation. If such a black hole absorbs an infalling neutral particle, the hole's mass will be increased while the charge remains fixed. This lowers the charge-to-mass ratio and raises the temperature of the black hole above zero. The black hole then emits one or several particles, and thereby loses enough mass to return to its stable extremal ground state. Viewed from afar, this appears to be a scattering process: there is a well defined incoming state (the infalling particle) and a well defined outgoing state (the created particles). The most powerful tools of quantum field theory, which are explicitly designed to deal with perturbative scattering, may then be brought to the study of this situation.
Within the context of this model, Callan et al. found that black holes did not form at all; the collapsing matter radiated away energy at a sufficient rate to prevent the formation of an event horizon. If an event horizon never forms, then the evolution of the quantum mechanical wavefunction from the past to the future remains deterministic.

On realizing that tractable twodimensional models of black-hole evaporation exist, quantum theorists have rapidly pressed them into service. Of the immense amount of new work, most has focused on understanding the possible physics of black-hole remnants, the massive $\left(10^{-5}\right.$ gram $)$ objects, $10^{-33} \mathrm{~cm}$ in size, which may contain all the information in the original collapsing star. If such remnants exist, they are surely the most compact information storage containers in nature. A model of such a remnant consisting of a tiny extreme charged black hole has been termed a 'cornucopion' because of the need to have a tremendous amount of information stored in an incredibly small object ${ }^{6}$.

What then is the present state of our knowledge concerning the endpoint of black-hole evaporation? As the twodimensional studies progress, the possibility that the information in the collapsing matter escapes through subtle correlations in the Hawking radiation seems increasingly unlikely. Further insights into the behaviour of remnants are emerging; however, there are still serious difficulties concerning possible copious pair creation of these objects as fundamental particles. Finally, Hawking's original notion that the information is in fact destroyed by black-hole evaporation remains a real possibility, although one that will not be palatable to many theoretical physicists until other possible resolutions are completely ruled out.

Since its discovery, black-hole evaporation has been the process whose theoretical study has provided the greatest insights into the physical nature of quantum gravity. The present enthusiasm for this field results from the realization that out of the apparent crisis in theoretical physics posed by information loss in black holes there may arise a new understanding of physics at the most fundamental levels.

William A. Hiscock is in the Department of Physics, Montana State University, Bozeman, Montana 59717, USA

\footnotetext{
1. Hawking, S. W. Nature 248, 30 (1974); Commun. math Phys. 43, 199-220 (1975)

Callan, C. G., Giddings, S. B., Harvey, J. A. \& Strominger, A. Phys. Rev. D45, R1005-R1009(1992).

3. Wald, R. General Relativity, 322-324 (University of Chicago Press, 1984)

4. Hawking, S. W. Phys. Rev. D14, 2460-2473 (1976).

5. Davies, P. C. W., Fulling, S. A. \& Unruh, W. G. Phys. Rev. D13, 2720-2723 (1976).

6. Banks, T., Dabholkar, A., Douglas, M. R. \& O'Loughlin, M. Phys. Rev. D45, 3607-3616 (1992)
} 